\title{
Erratum: Spin structure of heavy-quark hybrids [Phys. Rev. D 99, 014017 (2019)]
}

\author{
Nora Brambilla, Wai Kin Lai, Jorge Segovia, Jaume Tarrús Castellà@, and Antonio Vairo
}

(Received 14 February 2020; published 13 May 2020)

DOI: 10.1103/PhysRevD.101.099902

In the previously published version of the paper, in Eq. (6) the terms in the operator with coefficient $V_{\mathrm{SK} b}(r)$ had a wrong relative sign. Also, in Eq. (7) an operator of the form $\left[\left(\boldsymbol{r} \cdot \boldsymbol{P}_{1 \lambda}^{\dagger}\right)(\boldsymbol{p} \times \boldsymbol{S}) \cdot \boldsymbol{P}_{1 \lambda^{\prime}}+\boldsymbol{P}_{1 \lambda}^{\dagger} \cdot(\boldsymbol{p} \times \boldsymbol{S})\left(\boldsymbol{r} \cdot \boldsymbol{P}_{1 \lambda^{\prime}}\right)\right]$ was missing. In order to correct these two points, the following changes in the paper should be made.

(a) In Eq. (6), in the operator multiplying $V_{\mathrm{SK} b}(r)$, the sign of the second term should be (i) instead of + , i.e., the correct equation should be

$$
V_{1^{+-\lambda \lambda^{\prime} \mathrm{SD}}}^{(1)}(\boldsymbol{r})=V_{\mathrm{SK}}(r)\left(P_{1 \lambda}^{i \dagger} \boldsymbol{K}^{i j} P_{1 \lambda^{\prime}}^{j}\right) \cdot \boldsymbol{S}+V_{\mathrm{SK} b}(r)\left[\left(\boldsymbol{r} \cdot \boldsymbol{P}_{1 \lambda}^{\dagger}\right)\left(r^{i} \boldsymbol{K}^{i j} P_{1 \lambda^{\prime}}^{j}\right) \cdot \boldsymbol{S}-\left(r^{i} \boldsymbol{K}^{i j} P_{1 \lambda}^{j \dagger}\right) \cdot \boldsymbol{S}\left(\boldsymbol{r} \cdot \boldsymbol{P}_{1 \lambda^{\prime}}\right)\right]+\ldots
$$

(b) In Eq. (7), one operator is missing, which has coefficient $V_{\mathrm{SL} c}$. The correct equation should be

$$
\begin{aligned}
V_{1^{+-\lambda \lambda^{\prime} \mathrm{SD}}}^{(2)}(\boldsymbol{r})= & V_{\mathrm{SL} a}(r)\left(P_{1 \lambda}^{i \dagger} \boldsymbol{L}_{Q \bar{Q}} P_{1 \lambda^{\prime}}^{i}\right) \cdot \boldsymbol{S}+V_{\mathrm{SL} b}(r) P_{1 \lambda}^{i \dagger}\left(L_{Q \bar{Q}}^{i} S^{j}+S^{i} L_{Q \bar{Q}}^{j}\right) P_{1 \lambda^{\prime}}^{j}+V_{\mathrm{SL} c}(r)\left[\left(\boldsymbol{r} \cdot \boldsymbol{P}_{1 \lambda}^{\dagger}\right)(\boldsymbol{p} \times \boldsymbol{S}) \cdot \boldsymbol{P}_{1 \lambda^{\prime}}\right. \\
& \left.+\boldsymbol{P}_{1 \lambda}^{\dagger} \cdot(\boldsymbol{p} \times \boldsymbol{S})\left(\boldsymbol{r} \cdot \boldsymbol{P}_{1 \lambda^{\prime}}\right)\right]+V_{\mathrm{S}^{2}}(r) \boldsymbol{S}^{2} \delta_{\lambda \lambda^{\prime}}+V_{\mathrm{S}_{12} a}(r) S_{12} \delta_{\lambda \lambda^{\prime}}+V_{\mathrm{S}_{12} b}(r) P_{1 \lambda}^{i \dagger} P_{1 \lambda^{\prime}}^{j}\left(S_{1}^{i} S_{2}^{j}+S_{2}^{i} S_{1}^{j}\right)+\ldots
\end{aligned}
$$

Therefore, at order $1 / \mathrm{m}^{2}$, three new operators appear involving the projectors associated to the representations of $D_{\infty h}$, besides the usual spin-orbit, spin-spin, and tensor operators familiar from the studies of standard quarkonia.

(c) With the addition of the new operator with coefficient $V_{\mathrm{SL} c}$, the equation

$$
V_{\mathrm{SL} c}=V_{\mathrm{SL} c}^{\mathrm{np}}
$$

should be added to the set of equations Eqs. (10)-(16).

(d) On Page 4, the sentence "The term proportional to $V_{\mathrm{SK} b}$ contributes only to off-diagonal terms $\left(\lambda \neq \lambda^{\prime}\right)$ and turns out to be therefore negligible for the present computation." should be eliminated. The sentence "The size of the other

TABLE I. Nonperturbative matching coefficients determined by comparing the charmonium hybrid spectrum obtained from the hybrid EFT to the lattice spectrum

\begin{tabular}{|c|c|}
\hline$V_{\mathrm{SK}}^{\mathrm{np}(0)} / \Lambda_{\mathrm{QCD}}^{2}$ & 1.03 \\
\hline$V_{\mathrm{SK}}^{\mathrm{np}(1)} / \Lambda_{\mathrm{QCD}}^{4}$ & -0.51 \\
\hline$V_{\mathrm{SK} b}^{\mathrm{np}(0)} / \Lambda_{\mathrm{QCD}}^{4}$ & 0.28 \\
\hline$V_{\mathrm{SL} a}^{\mathrm{np}(0)} / \Lambda_{\mathrm{QCD}}^{3}$ & -1.32 \\
\hline$V_{\mathrm{SL} b}^{\mathrm{np}(0)} / \Lambda_{\mathrm{QCD}}^{3}$ & 2.44 \\
\hline$V_{\mathrm{SL} c}^{\mathrm{np}(0)} / \Lambda_{\mathrm{QCD}}^{3}$ & 0.87 \\
\hline$V_{\mathrm{S}^{2}}^{\mathrm{np}(0)} / \Lambda_{\mathrm{QCD}}^{3}$ & -0.33 \\
\hline$V_{\mathrm{S}_{12} b}^{\mathrm{np}(0)} / \Lambda_{\mathrm{QCD}}^{3}$ & -0.39 \\
\hline
\end{tabular}
from the Hadron Spectrum Collaboration data of Ref. [34] with pion mass of $m_{\pi} \approx 240 \mathrm{MeV}$, respectively. The matching coefficients are normalized to their parametric natural size. We take the value $\Lambda_{\mathrm{QCD}}=0.5 \mathrm{GeV}$.

Published by the American Physical Society under the terms of the Creative Commons Attribution 4.0 International license. Further distribution of this work must maintain attribution to the author(s) and the published articles title, journal citation, and DOI. 

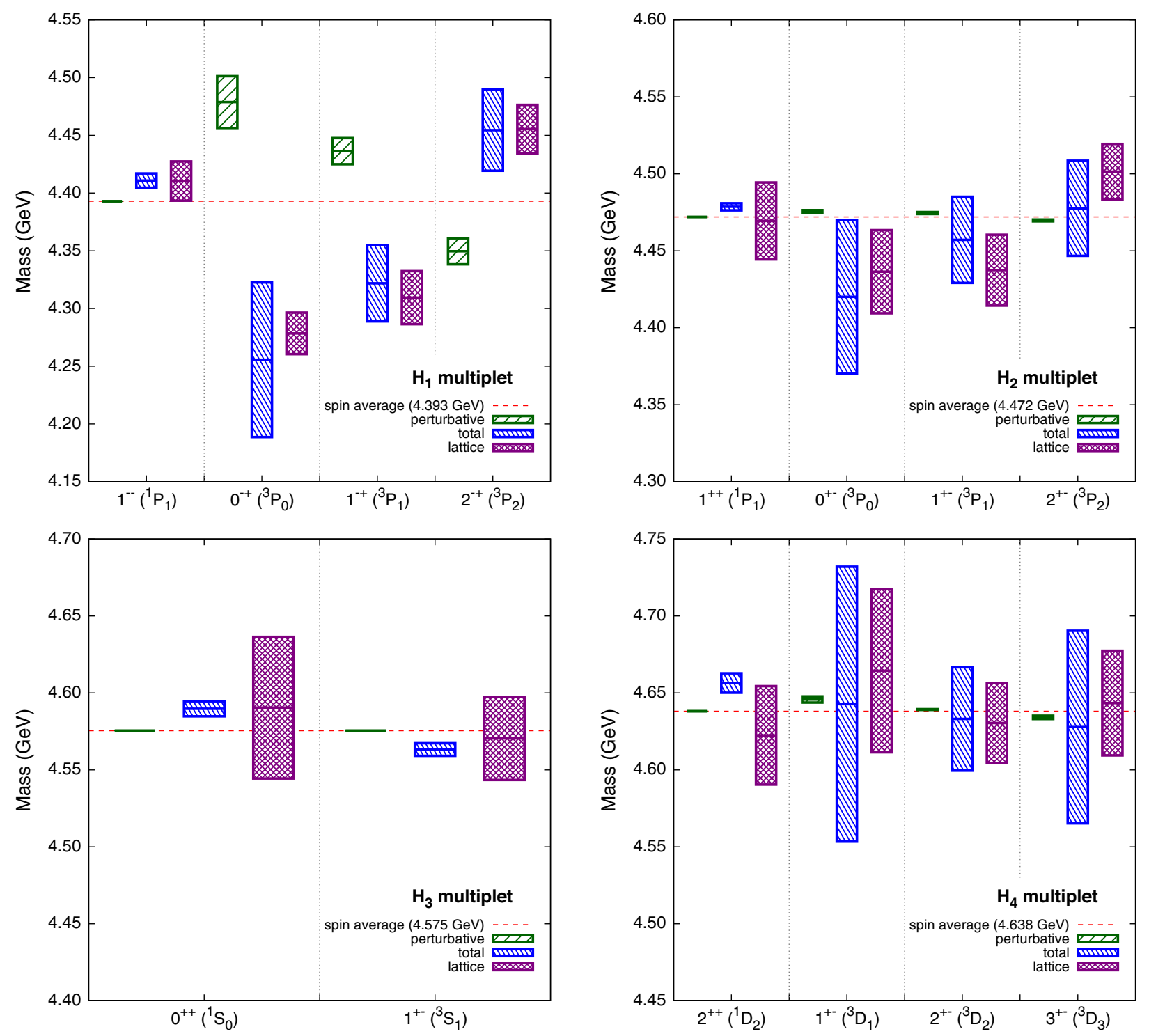

FIG. 1. Spectrum of the four lowest-lying charmonium hybrid multiplets. The lattice results from Ref. [34] with $m_{\pi} \approx 240 \mathrm{MeV}$ are the most right (purple) boxes for each quantum number. The perturbative contributions to the spin-dependent operators in Eq. (7) added to the spin average of the lattice results (red dashed lines) are the most left (green) boxes. The central (blue) boxes for each quantum number are the full results from the spin-dependent operators of Eqs. (6) and (7) including perturbative and nonperturbative contributions. The unknown nonperturbative matching coefficients are determined through a fitting procedure that reproduces the lattice data. The height of the boxes indicates the uncertainty as detailed in the text.

potentials follows from dimensional analysis: $V_{\mathrm{SL} a}^{\mathrm{np}(0)}, V_{\mathrm{SL} b}^{\mathrm{np}(0)}, V_{\mathrm{S}^{2}}^{\mathrm{np}(0)}, V_{\mathrm{S}_{12} b}^{\mathrm{np}(0)} \sim \Lambda_{\mathrm{QCD}}^{3}$ " should be modified to "The size of the other potentials follows from dimensional analysis: $V_{\mathrm{SL} a}^{\mathrm{np}(0)}, V_{\mathrm{SL} b}^{\mathrm{np}(0)}, V_{\mathrm{SL} c}^{\mathrm{np}(0)}, V_{\mathrm{S}^{2}}^{\mathrm{np}(0)}, V_{\mathrm{S}_{12} b}^{\mathrm{np}(0)} \sim \Lambda_{\mathrm{QCD}}^{3}$, and $V_{\mathrm{SK} b}^{\mathrm{np}(0)} \sim \Lambda_{\mathrm{QCD}}^{4} . "$

(e) On page 5, the sentence "The six nonperturbative parameters $V_{\mathrm{SK}}^{\mathrm{np}(0)}, V_{\mathrm{SK}}^{\mathrm{np}(1)}, V_{\mathrm{SL} a}^{\mathrm{np}(0)}, V_{\mathrm{SL} b}^{\mathrm{np}(0)}, V_{\mathrm{S}^{2}}^{\mathrm{np}(0)}, V_{\mathrm{S}_{12} b}^{\mathrm{np}(0)}$ that appear in the spin-dependent potentials in Eqs. (10)-(16) are obtained by fitting the spin splittings resulting from our calculation to lattice data of the charmonium hybrid spectrum." should be modified to "The eight nonperturbative parameters $V_{\mathrm{SK}}^{\mathrm{np}(0)}, V_{\mathrm{SK}}^{\mathrm{np}(1)}, V_{\mathrm{SK} b}^{\mathrm{np}(0)}, V_{\mathrm{SL} a}^{\mathrm{np}(0)}, V_{\mathrm{SL} b}^{\mathrm{np}(0)}, V_{\mathrm{SL} c}^{\mathrm{np}(0)}, V_{\mathrm{S}^{2}}^{\mathrm{np}(0)}, V_{\mathrm{S}_{12} b}^{\mathrm{np}(0)}$ that appear in the spin-dependent potentials in Eqs (10)-(16) are obtained by fitting the spin splittings resulting from our calculation to lattice data of the charmonium hybrid spectrum." 

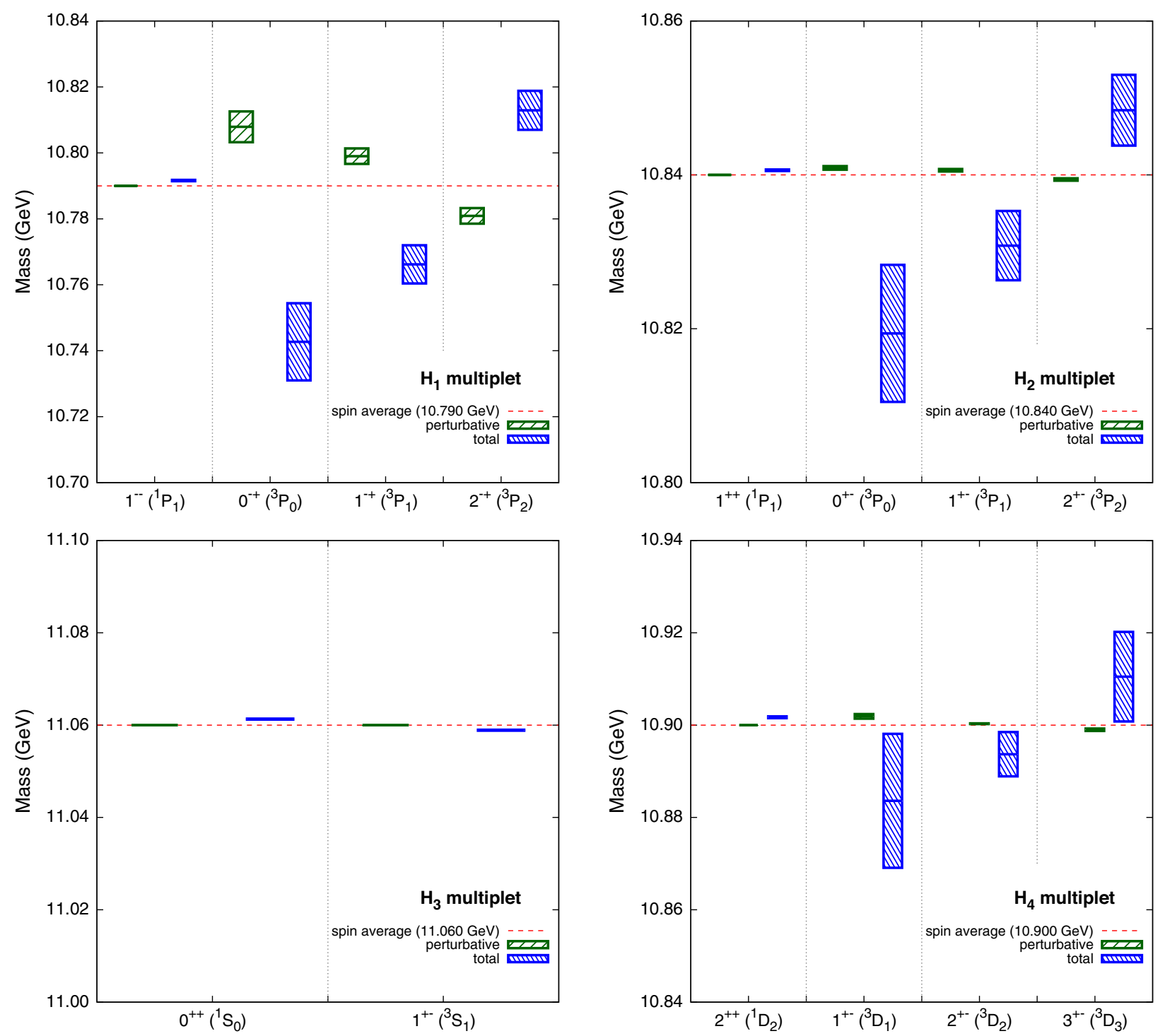

FIG. 2. Spectrum of the four lowest-lying bottomonium hybrids computed by adding the spin-dependent contributions from Eqs. (10)-(16) to the spectrum obtained in Ref. [44]. The nonperturbative contribution to the matching coefficients is determined from the charmonium hybrids spectrum of Ref. [34] shown in Fig. 2. The average mass for each multiplet is shown as a red line. The results with only the perturbative contributions included and the full results with perturbative and nonperturbative contributions included are shown for each quantum number by the left (green) and the right (blue) boxes, respectively. The height of the boxes indicates the uncertainty as detailed in the text.

(f) Table II is modified as included below (Table I). Note that two new entries are added for $V_{\mathrm{SK} b}^{\mathrm{np}}$ and $V_{\mathrm{SL} c}^{\mathrm{np}(0)}$. In obtaining the numbers in Table II, we adopt the following procedures: We use the values of $V_{\mathrm{SK}}^{\mathrm{np}(0)}, V_{\mathrm{SK}}^{\mathrm{np}(1)}, V_{\mathrm{SL} a}^{\mathrm{np}(0)}, V_{\mathrm{SL} b}^{\mathrm{np}(0)}, V_{\mathrm{S}^{2}}^{\mathrm{np}(0)}$, $V_{\mathrm{S}_{12} b}^{\mathrm{np}(0)}$ determined in the previous version of the paper, which correspond to fitting to the lattice data of the charmonium hybrid spectrum with $V_{\mathrm{SK} b}^{\mathrm{np}(0)}$ and $V_{\mathrm{SL} c}^{\mathrm{np}(0)}$ set to zero. With these value fixed, we determine the values of $V_{\mathrm{SK} b}^{\mathrm{np}(0)}$ and $V_{\mathrm{SL} c}^{\mathrm{np}(0)}$ by fitting to the same set of lattice data. The resulting $\chi^{2} /$ d.o.f. for the eight $V^{\text {np (i) }}$ 's is 0.99998 . Note that the leading contribution $V_{\mathrm{SK}}^{\mathrm{np}(0)}$ has the most dominant effect. Since the parameter space is quite large and the uncertainty of the lattice data is comparable to the size of the subleading contributions, the values of the $V^{\mathrm{np}(\mathrm{i})}$ 's other than $V_{\mathrm{SK}}^{\mathrm{np}(0)}$ cannot be reliably determined.

(g) Figures 2 and 3 should be replaced by the new figures below (Figs. 1 and 2). Note that the essential features of the figures are not changed.

(h) In Eqs. (A8) and (A9), $\partial_{\theta}$ should be replaced by $\partial_{\phi}$. 